\title{
Anti-embolic stockings (AES) for cardiac surgery patients in the peri- operative period.
}

\author{
Mohammad Miah', Mauin Uddin²*, Mohammad Parvez Ahmed ${ }^{3}$, Syed Al Nahian, Thomas Seddon ${ }^{1}$, \\ Ahmed Ashoub ${ }^{1}$
}

${ }^{1}$ Queen Elizabeth Hospital, Birmingham, United Kingdom

${ }^{2}$ New Cross Hospital, Wolverhampton, United Kingdom

${ }^{3}$ University Hospital Coventry and Warwickshire, Coventry CV2 2DX, United Kingdom

${ }^{4}$ Liverpool Heart and Chest Hospital, Liverpool L14 3PE, United Kingdom

\begin{abstract}
The inconsistent use of prophylactic measures for VTE in hospital patients has been widely reported. A UK survey suggested that $71 \%$ of patients assessed to be at medium or high risk of developing deep vein thrombosis did not receive any form of mechanical or pharmacological VTE prophylaxis. All patients to be assessed on admission to identify those who are at increased risk of VTE. Our audit objective was to find out any perioperative period in which patient was not on AES, if there was any. Consecutive 300 patients underwent Cardiac Surgery between August 2017 and December 2017 at Queen Elizabeth Hospital Birmingham were included. Vast majority of the patients were found more than 5 days without AES. More wide use of VTE prophylaxis, early mobilization, and better perioperative care have reduced the incidence of VTE in surgical patients.
\end{abstract}

Keywords: Anti-embolic stockings, Cardiac surgery.

Accepted on May 06, 2020

\section{Background}

The House of Commons Health Committee [1] reported in 2005 that an estimated 25,000 people in the UK die from preventable hospital acquired venous thromboembolism (VTE) every year. This includes patients admitted to hospital for medical care and surgery. The inconsistent use of prophylactic measures for VTE in hospital patients has been widely reported. A UK survey suggested that $71 \%$ of patients assessed to be at medium or high risk of developing deep vein thrombosis did not receive any form of mechanical or pharmacological VTE prophylaxis [2]. All patients to be assessed on admission to identify those who are at increased risk of VTE. All patients should be assessed for risk of bleeding before offering pharmacological VTE prophylaxis [3-11]. Pharmacological VTE prophylaxis should not be offered to patients with any of the risk factors for bleeding (shown in box 1) unless the risk of VTE outweighs the risk of bleeding. Patients' risks of bleeding and VTE to be reassessed within 24 hours of admission and whenever the clinical situation changes, to ensure that the methods of VTE prophylaxis being used are suitable, used correctly and identify adverse events resulting from VTE prophylaxis.

\section{Assessing the Risks of VTE and Bleeding}

\section{VTE risk factors}

Regard surgical patients and patients with trauma as being at increased risk of VTE if they meet one of the following criteria:
Surgical procedure with a total anaesthetic and surgical time of more than 90 minutes, or 60 minutes if the surgery involves the pelvis or lower limb.

Acute surgical admission with inflammatory or intra-abdominal condition.

Expected significant reduction in mobility.

One or more of the risk factors shown in box 1.

\section{Box: 1}

Active cancer or cancer treatment.

Age over 60 years, Obesity (body mass index [BMI] over $30 \mathrm{~kg} / \mathrm{m}^{2}$ ).

Critical care admission.

Dehydration.

Known thrombophilias.

One or more significant medical comorbidities (for example: heart disease; metabolic, endocrine or respiratory pathologies; acute infectious diseases; inflammatory conditions).

Personal history or firstdegree relative with a history of VTE.

Use of hormone replacement therapy, oestrogencontaining contraceptive therapy.

Varicose veins with phlebitis.

For women who are pregnant or have given birth within the previous 6 weeks.

Curr Trend Cardiol 2020 Volume 4 Issue 1 
Citation: Miah M, Uddin M, Ahmed MP, et al. Anti-embolicstockings (AES) for cardiac surgery patients in the peri-operative period. Curr Trend Cardiol 2020;4(1):1-3.

\section{Risk factors for bleeding}

Active bleeding.

Acquired bleeding disorders (such as acute liver failure).

Concurrent use of anticoagulants known to increase the risk of bleeding (such as warfarin with international normalised ratio [INR] higher than 2).

Lumbar puncture/epidural/spinal anaesthesia expected within the next 12 hours.

Lumbar puncture/epidural/spinal anaesthesia within the previous 4 hours.

Acute stroke.

Thrombocytopenia (platelets less than $75 \times 109 / 1$ ).

Uncontrolled systolic hypertension (230/120 mmHg or higher).

Untreated inherited bleeding disorders (such as haemophilia and von Willebrand's disease).

\section{Mechanical VTE Prophylaxis}

Base the choice of mechanical VTE prophylaxis on individual patient factors including clinical condition, surgical procedure and patient preference. Choose any one of:

Anti-embolism stockings (AES), thigh or knee length.

Foot impulse devices.

Intermittent pneumatic compression devices (thigh or knee length).

\section{AntiEmbolism Stockings (AES):}

\section{Contraindications}

Do not offer antiembolism stockings to patients who have:

Suspected or proven peripheral arterial disease.

Peripheral arterial bypass grafting.

Peripheral neuropathy or other causes of sensory impairment.

Any local conditions in which stockings may cause damage, for example fragile 'tissue paper' skin, dermatitis, gangrene or recent skin graft.

Known allergy to material of manufacture.

Cardiac failure.

Severe leg oedema or pulmonary oedema from congestive heart failure.

Major limb deformity preventing correct fit.
Use caution and clinical judgement when applying antiembolism stockings over venous ulcers or wounds.

\section{Method and Sample}

Our audit objective was to find out any perioperative period in which patient was not on AES, if there was any. Retrospective

data were collected via PICs system. Consecutive 300 patients underwent Cardiac Surgery between August 2017 and December 2017 at Queen Elizabeth Hospital Birmingham were included. Patients were excluded if they had contraindications to AES.

\section{Results}

Vast majority of the patients were found more than 5 days without AES. In one third of the patients there was a significant delay of 3 days between the time of AES prescribed and received. Staggering number of postoperative patients were found more than 3 days in the ward without AES (Figures 1-3).

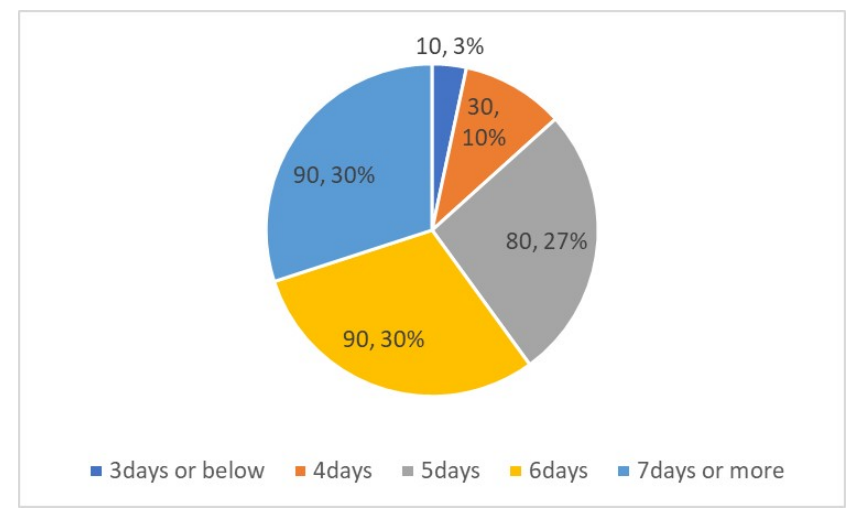

Figure 1. Pie chart showing days without AES.

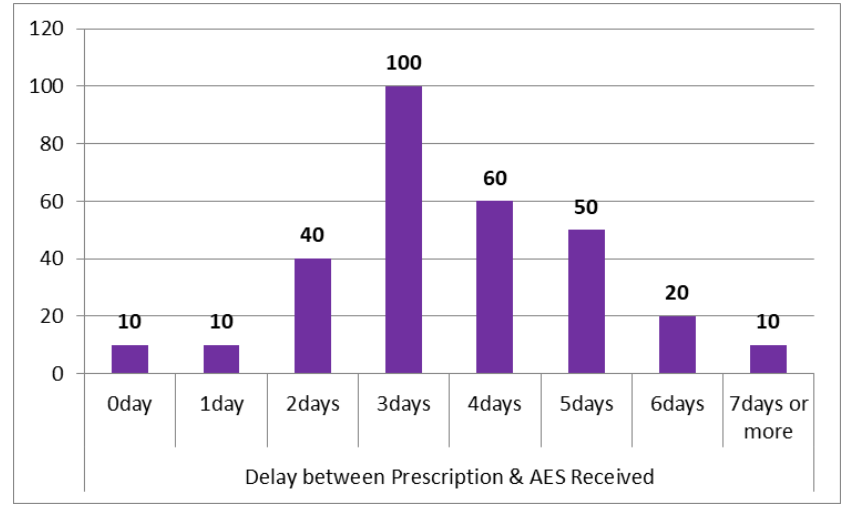

Figure 2. Bar graph showing the delay between prescription and AES received in various number of patients. 


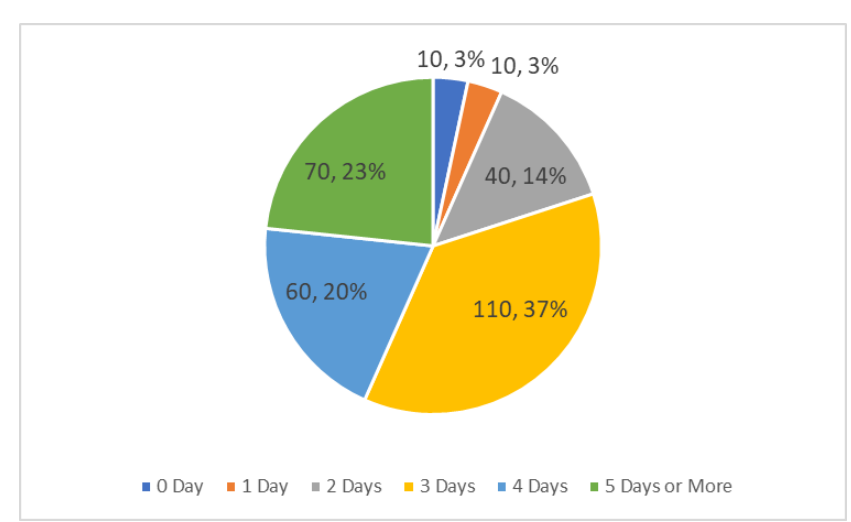

Figure 3. Pie Chart showing the patients without AES in ward at different postoperative periods.

\section{Discussion and Conclusion}

VTE is a common adverse event in patients undergoing surgery. Pulmonary embolism PE is the most common cause of preventable death in patients following surgical procedures. More wide use of VTE prophylaxis, early mobilization, and better peri-operative care have reduced the incidence of VTE in surgical patients. However, many patients remain at high risk for VTE because of advanced age, more complicated operative procedures, and increased medical co-morbidities. Postoperative DVT of the lower limbs is often asymptomatic; in many patients; fatal PE is the first clinical manifestation of postoperative VTE. For that, it is inappropriate to rely on early diagnosis and treatment of postoperative VTE. If applied carefully, such prophylaxis is cost effective because it reduces the incidence of symptomatic thromboembolic complications, which require costly diagnostic methods and lengthy anticoagulation therapy.

\section{References}

1. The prevention of venous thromboembolism in hospitalised patients. London: The Stationery Office. House of Commons Health Committee 2005.

2. Rashid ST, Thursz MR, Razvi NA, et al. Venous thromboprophylaxis in UK medical inpatients. J R Soc Med. 2005; 98:507-12.
3. Thomas H, Diamond J, Vieco A, et al. Global Atlas of Cardiovascular Disease 2000-2016: The Path to Prevention and Control. Glob Heart. 2018; 13(3):143-63.

4. Heidenreich PA, Trogdon JG, Khavjou OA, et al. Forecasting the future of cardiovascular disease in the United States: a policy statement from the American Heart Association. Circulation. 2011; 123(8):933-44.

5. Corella D, Asensio EM, Coltell O, et al. CLOCK gene variation is associated with incidence of type- 2 diabetes and cardiovascular diseases in type-2 diabetic subjects: dietary modulation in the PREDIMED randomized trial. CardiovascDiabetol. 2016; 15:4.

6. Mormile R. Multiple sclerosis and susceptibility to cardiovascular diseases: Implications of ethnicity-related interleukin-17A gene polymorphism? Med Hypotheses. 2015; 85(3):365-66.

7. Pasipoularides A. Linking genes to cardiovascular diseases: Gene action and gene-environment interactions. J Cardiovasc Transl Res. 2015; 8(9):506-27.

8. Kim YG, Cha J, Chandrasegaran S. Hybrid restriction enzymes: Zinc finger fusions to Fok I cleavage domain. Proc Natl AcadSci USA. 1996; 93(3):1156-60.

9. Jiang W, Bikard D, Cox D, et al. RNA-guided editing of bacterial genomes using CRISPR-Cas systems. Nat Biotechnol. 2013; 31(3):233-39.

10. Gao C. The future of CRISPR technologies in agriculture. Nat Rev Mol Cell Biol. 2018; 19(5):275-76.

11. Fellmann C, Gowen BG, Lin PC, et al. Cornerstones of CRISPR-Cas in drug discovery and therapy. Nat Rev Drug Discov. 2017;16(2):89-100.

\section{*Correspondence to}

Mauin Uddin

New Cross Hospital

Wolverhampton

United Kingdom

E-mail:mu_ahmed07@yahoo.com 